\title{
Efficacy of Rg1-Oil Adjuvant on Inducing Immune Responses against Bordetella bronchiseptica in Rabbits
}

\author{
Xiao Chenwen, ${ }^{1}$ Ji Quanan, ${ }^{1}$ Huang Yee, ${ }^{1}$ Liu Yan, ${ }^{1}$ Wang Jiaoyu, ${ }^{2}$ Wei Qiang, ${ }^{1}$ Qiao Litao, ${ }^{3}$ \\ Nan Li, ${ }^{1}$ and Bao Guolian (iD) ${ }^{1}$ \\ ${ }^{1}$ Institute of Animal Husbandry and Veterinary Science, Zhejiang Academy of Agricultural Sciences, Hangzhou, \\ Zhejiang 310021, China \\ ${ }^{2}$ Institute of Plant Protection and Microbiology, Zhejiang Academy of Agricultural Science, Hangzhou 310021, China \\ ${ }^{3}$ Department of Ultrasound, PKUCare Luzhong Hospital, Zibo, Shandong 255400, China \\ Correspondence should be addressed to Bao Guolian; baoguolian2020@163.com
}

Received 19 September 2020; Revised 17 November 2020; Accepted 20 January 2021; Published 29 January 2021

Academic Editor: Paulina Niedźwiedzka-Rystwej

Copyright ( 92021 Xiao Chenwen et al. This is an open access article distributed under the Creative Commons Attribution License, which permits unrestricted use, distribution, and reproduction in any medium, provided the original work is properly cited.

Bordetella bronchiseptica (B. bronchiseptica) is an obligately aerobic, oxidase- and catalase-positive, nonfermentative Gramnegative coccobacillus. This study is aimed at examining the immune effects of Rg1, Rg1 plus oil, and other common adjuvants on inactivated B. bronchiseptica vaccine in rabbits. The mechanism underlying the adjuvant effect of $\operatorname{Rg} 1$ plus oil on the vaccine was also explored. $\operatorname{Rg} 1(100 \mu \mathrm{g})$ plus oil significantly improved the immune effect of $B$. bronchiseptica vaccine at both the humoral and cellular levels. Rg1-oil adjuvant increased the levels of IL-2 and IL-4 in rabbits after immunization. Rg1 (100 $\mu \mathrm{g}$ ) plus oil also significantly increased TLR2 expression and downregulated NF- $\kappa \mathrm{B}$ in splenocytes. Rg1-oil adjuvant may increase the levels of IL-2 and IL-4 via upregulating TLR2, thereby enhancing the immune effect of B. bronchiseptica vaccine. In conclusion, Rg1 plus oil could be used as a potential vaccine adjuvant for rabbit B. bronchiseptica vaccine.

\section{Introduction}

Bordetella bronchiseptica (B. bronchiseptica) is an obligately aerobic, oxidase- and catalase-positive, nonfermentative Gram-negative coccobacillus that was first identified in dogs with distemper disease in 1911 [1]. It causes infections in the respiratory tract of rabbits and acts as a precursor for secondary infection with Pasteurella multocida [2]. B. bronchiseptica infection in rabbits occurs all year round, but more commonly in autumn and spring. The nasal secretion from infected animals may contaminate air, food, and water, which facilitates the spread of the disease. B. bronchiseptica infection not only induces pustular pneumonia, bronchitis, and rhinitis in adult rabbits but also leads to acute death in young animals both before and after weaning. The rapid spread and difficulties in complete eradication of this disease often result in serious economic loss [3].

Vaccination is one of the most effective interventions to prevent $B$. bronchiseptica infection in rabbits. However, the development of adjuvants for $B$. bronchiseptica vaccine is still challenging. Currently available adjuvants, such as synthetic peptide vaccine and DNA recombinant vaccine, do not meet the demands of vaccines with low immunogenicity. Extensive research on the development of novel adjuvants has been carried out. However, these adjuvants have not been widely used due to poor safety performance. Significant advances have been made to enhance the potency of currently available vaccines by incorporating adjuvants into vaccine formulations. Besides the immune-stimulating effect, adjuvants also reduce the amount of antigens needed for vaccination, thus reducing the cost of production. However, the incorporation of adjuvants into the vaccine should be carefully evaluated to ensure that no adverse side effects occur and the immunogenic effect is stimulated [4]. Ginseng, the root of Panax ginseng C.A. Meyer (Araliaceae), is a herbal tonic used in traditional Chinese medicine for over 2000 years. The adjuvant effects of ginseng saponins (ginsenosides) on immune responses in rats, mice, pigs, and cattle have been widely reported [5]. 
TABle 1: Adjuvant vaccines used in Experiment A.

\begin{tabular}{lccc}
\hline No. & Groups & Preparation each dose \\
\hline 1 & 1 & Rg1 $(400 \mu \mathrm{g})$ in PBS $500 \mu \mathrm{L}$ & $1.5 \times 10^{10} \mathrm{CFU}$ in PBS $500 \mu \mathrm{L}$ \\
2 & 2 & $750 \mu \mathrm{L}$ oil $+10 \mu \mathrm{L}$ Tween + Rgl $(400 \mu \mathrm{g})$ & $1.5 \times 10^{10} \mathrm{CFU}$ in PBS $500 \mu \mathrm{L}$ \\
3 & 3 & $750 \mu \mathrm{L}$ oil $+10 \mu \mathrm{L}$ Tween & $1.5 \times 10^{10} \mathrm{CFU}$ in PBS $500 \mu \mathrm{L}$ \\
4 & 4 & $50 \mu \mathrm{g} \mathrm{QA}$ in PBS $500 \mu \mathrm{L}$ & $1.5 \times 10^{10} \mathrm{CFU}$ in PBS $500 \mu \mathrm{L}$ \\
5 & 5 & Alum $(200 \mu \mathrm{g})$ in PBS $500 \mu \mathrm{L}$ & $1.5 \times 10^{10} \mathrm{CFU}$ in PBS $500 \mu \mathrm{L}$ \\
6 & 6 & No adjuvant & $1.5 \times 10^{10} \mathrm{CFU}$ in PBS $500 \mu \mathrm{L}$ \\
\hline
\end{tabular}

TABle 2: Adjuvant vaccines used in Experiment B.

\begin{tabular}{lccc}
\hline No. & Groups & Preparation each dose \\
\hline 1 & Rg1+oil & $750 \mu \mathrm{L}$ oil $+10 \mu \mathrm{L}$ Tween + Rg1 $(100 \mu \mathrm{g})$ & $1.5 \times 10^{10} \mathrm{CFU}$ in PBS $250 \mu \mathrm{L}$ \\
2 & Rg1+oil & $750 \mu \mathrm{L}$ oil $+10 \mu \mathrm{L}$ Tween + Rg1 $(200 \mu \mathrm{g})$ & $1.5 \times 10^{10} \mathrm{CFU}$ in PBS $250 \mu \mathrm{L}$ \\
3 & Rg1+oil & $750 \mu \mathrm{L}$ oil $+10 \mu \mathrm{L}$ Tween + Rg1 $(400 \mu \mathrm{g})$ & $1.5 \times 10^{10} \mathrm{CFU}$ in PBS $250 \mu \mathrm{L}$ \\
4 & Oil & $750 \mu \mathrm{L}$ oil $+10 \mu \mathrm{L}$ Tween & $1.5 \times 10^{10} \mathrm{CFU}$ in PBS $250 \mu \mathrm{L}$ \\
5 & No adjuvant & $1.5 \times 10^{10} \mathrm{CFU}$ in PBS $250 \mu \mathrm{L}$ \\
6 & PBS & $1000 \mu \mathrm{L}$ & $1.5 \times 10^{10} \mathrm{CFU}$ in PBS $250 \mu \mathrm{L}$ \\
\hline
\end{tabular}

Rg1 is a ginsenoside that exhibits adjuvant activity in immune responses [6,7]. In this work, we evaluated the immune effects of Rg1 and other common adjuvants on inactivated rabbit $B$. bronchiseptica vaccine and explored the adjuvant mechanism of Rg1. Our findings provided scientific support for the development of $B$. bronchiseptica vaccines.

\section{Materials and Methods}

2.1. Antigens and Adjuvants. White oil (mineral oil), alhydrogel adjuvant, and $B$. bronchiseptica antigen were obtained from Connaught Times Wei Biotechnology Company (Hangzhou, China). B. bronchiseptica (FX strain) was cultured through GMP-certified fermentation followed by sterilization with $0.2 \%$ formalin for $36 \mathrm{~h}$. After a sterilization test, $B$. bronchiseptica was stored at $4^{\circ} \mathrm{C}$ for further analyses. Whole proteins were isolated from $B$. bronchiseptica for ELISA. Rg1 was purchased from Yuanye Biotechnology (Cat No. 22427-39-0, Shanghai, China). QA was kindly provided by Zhejiang University. Quil A was obtained from Desert King Chile Ltd. (Santiago, Chile). QA and Rg1 solution was analyzed for endotoxin using a Limulus Amebocyte Lysate gel-clot assay (Cat No. 118792, Zhanjiang A\&C Biological Ltd., Zhanjiang, China) to ensure that the endotoxin level was $<0.5$ endotoxin units $/ \mathrm{mL}$.

2.2. Vaccine Preparation. Oil-based adjuvants were emulsified for $90 \mathrm{~min}$ using an automatic fast-sample grinding instrument (JingXin Technology, Shanghai, China) with an emulsification index of 60 hertz. This procedure was repeated twice. The components of each vaccine are shown in Tables 1 and 2.

2.3. Animals and Study Design. This study was approved by the Ethics Committee of the Zhejiang Academy of Agricultural Sciences (ethics protocol no. 002762) and performed
TABLE 3: Sequences of primers used in RT-PCR.

\begin{tabular}{lc}
\hline Gene & \multicolumn{1}{c}{ Sequence } \\
\hline NF- $\kappa$ B2-F & CTGGGTGTCCTACACGTGAC \\
NF- $\kappa$ B2-R & GATGGGCTGGGAGATAACGG \\
TLR2-F & CGTGTCAGGTCAGTCAGCTT \\
TLR2-R & ACCCTCTGGTACTCCGTCTC \\
ACTB-F & GTGCTTCTAGGCGGACTGTT \\
ACTB-R & TCGGCCACATTGCAGAACTT \\
\hline
\end{tabular}

in accordance with the principles and guidelines of the Zhejiang Farm Animal Welfare Council of China. Five-week-old male New Zealand rabbits (weighing 1-1.5 kg) were obtained from the Zhejiang Animal Center and allowed to acclimate for one week before experiments.

2.3.1. Experiment $A$. Rabbits were randomly assigned into eight groups ( $n=15$ per group) and then immunized by subcutaneous injection of $1 \mathrm{~mL}$ of $B$. bronchiseptica $\left(1.5 \times 10^{10} \mathrm{CFU}\right)$ alone, B. bronchiseptica $\left(1.5 \times 10^{10} \mathrm{CFU}\right)$ dissolved in PBS containing $400 \mu \mathrm{g}$ Rg1, $400 \mu \mathrm{g}$ Rg1 plus $750 \mu \mathrm{L}$ oil, $750 \mu \mathrm{L}$ oil alone, $50 \mu \mathrm{g} \mathrm{QA}$, or $200 \mu \mathrm{g}$ Alum into the neck on Day 1 (Table 1). Blood samples were collected from the ear vein of each animal before and at 5, 10, 15, 21, and 35 days after injection and kept at $4^{\circ} \mathrm{C}$. The body weight of each animal was measured before and after immunization on Days $0,5,10,15$, and 35 . All rabbits were monitored for adverse drug reactions after immunization.

2.3.2. Experiment B. Rabbits were randomly divided into six groups ( $n=8$ per group) and then subcutaneously injected with $1 \mathrm{~mL}$ of $B$. bronchiseptica $\left(1.5 \times 10^{10} \mathrm{CFU}\right)$ alone, $B$. bronchiseptica $\left(1.5 \times 10^{10} \mathrm{CFU}\right)$ dissolved in PBS containing 100,200 , or $400 \mu \mathrm{g} \operatorname{Rg} 1$ plus $750 \mu \mathrm{L}$ oil, or $750 \mu \mathrm{L}$ oil alone into the neck on Day 1 (Table 2). Blood samples were 

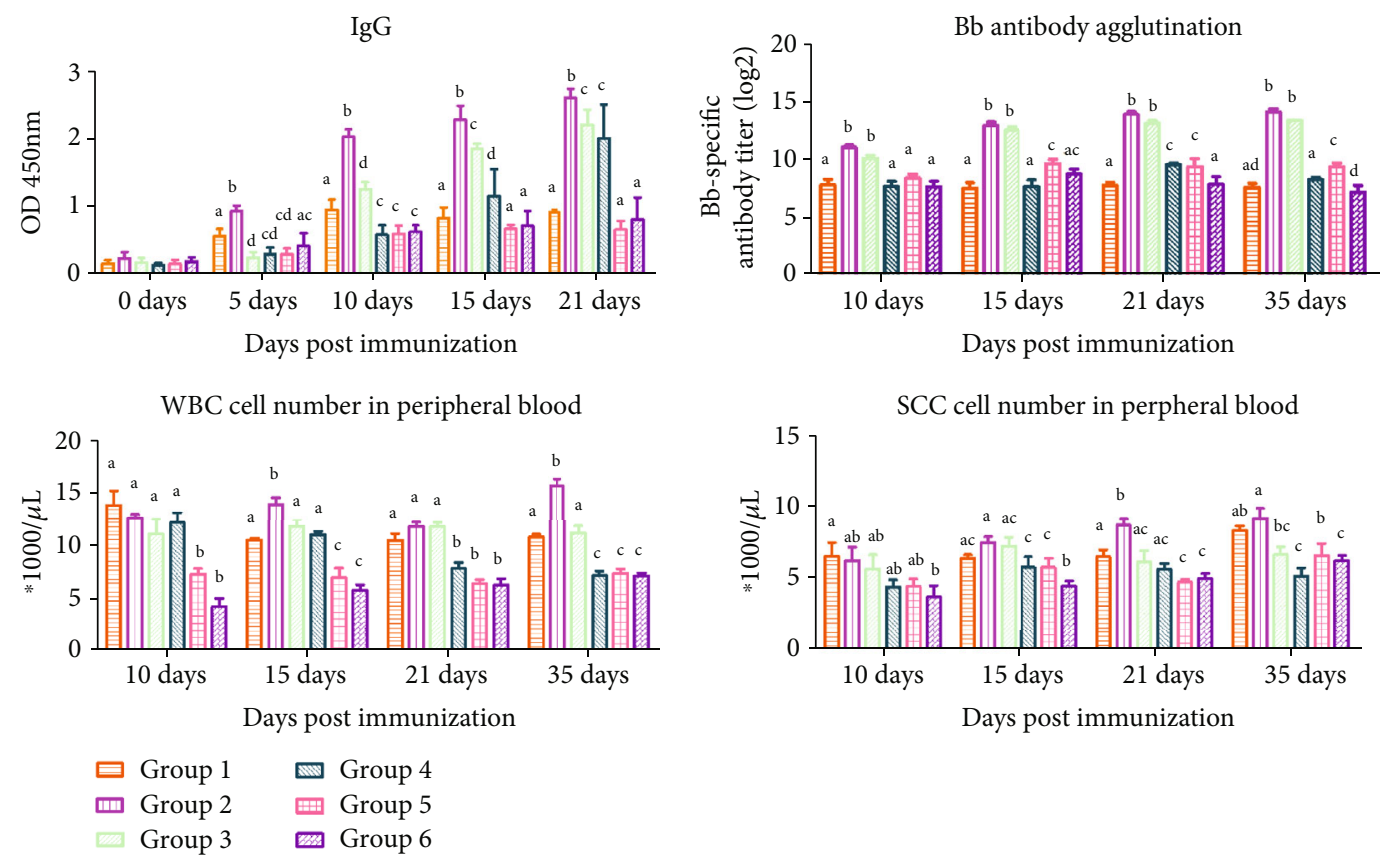

FIGURE 1: The serum level of whole bacterial protein-specific IgG antibody in rabbits from Experiment A was detected by indirect ELISA. An agglutination test was performed to measure B. bronchiseptica-specific antibody titer. Blood samples were collected from each group of rabbits as anticoagulants at 10,15,21, and 35 days postimmunization for the detection of WBC and SCC.

collected from the ear vein of each rabbit before immunization and at 15 and 30 days postinjection and stored at $4^{\circ} \mathrm{C}$. All rabbits were monitored for adverse drug reactions after immunization.

2.4. Measurement of the B. bronchiseptica-Specific IgG Level. The serum level of B. bronchiseptica-specific IgG was determined by indirect ELISA. Microtiter plate wells were purchased from Gongdong Medical Plastic Factory (Zhejiang, China). Each well was coated with $100 \mu \mathrm{L}$ of $1 \mu \mathrm{g} / \mathrm{mL} B$. bronchiseptica protein dissolved in $0.05 \mathrm{M}$ carbonate buffer overnight at $4^{\circ} \mathrm{C}$. After washing with $0.05 \%$ PBST (Tween20), wells were blocked with $5 \%$ nonfat milk for $2 \mathrm{~h}$ at $37^{\circ} \mathrm{C}$ followed by three washes. Then, $100 \mu \mathrm{L}$ diluted serum sample $(1: 400)$ was added to each well in triplicate. After $1 \mathrm{~h}$ incubation at $37^{\circ} \mathrm{C}$, a horseradish peroxidase-conjugated goat antirabbit antibody ( $1: 5000$, KLP, USA) was added to each well and incubated at $37^{\circ} \mathrm{C}$ for $1 \mathrm{~h}$. After washing with $0.05 \%$ PBST (Tween-20), samples were incubated with $100 \mu \mathrm{L}$ of substrate solution at $37^{\circ} \mathrm{C}$ for $10 \mathrm{~min}$. The reaction was terminated by the addition of $2 \mathrm{~N} \mathrm{H}_{2} \mathrm{SO}_{4}(50 \mu \mathrm{L}$ per well). The optical density (OD) was detected at $450 \mathrm{~nm}$ using an ELISA reader.

2.5. Analysis of the B. bronchiseptica-Specific Antibody Titer. Serum samples collected from Experiment A were serially diluted in PBS by twofold into a 12-well plate starting with a $1: 10$ dilution. Each well contained $50 \mu \mathrm{L}$ serum in a Vshaped 96-well plate. Subsequently, samples were gently mixed with $50 \mu \mathrm{L}$ of $B$. bronchiseptica (a mixture of $9 \mathrm{~mL} B$. bronchiseptica bacterial fluid (OD620 value $=2.5)$ and $100 \mu \mathrm{L}$ methylene blue) for $1 \mathrm{~h}$ at $37^{\circ} \mathrm{C}$ and then transferred to $4^{\circ} \mathrm{C}$. The next day, after $10 \mathrm{~min}$ incubation at room temperature, the test results were recorded, including blank (PBS), negative, and positive controls.

2.6. Analysis of Cytokine Levels. The levels of IL-2 and IL-4 in rabbits from Experiment B were determined by ELISA (Cat Nos. ml0029781-2 and ml 027170, MLBIO, Shanghai Enzyme-Linked Biotechnology, Shanghai, China). Each well was filled with $50 \mu \mathrm{L}$ of sample or standard and then added with $100 \mu \mathrm{L}$ of horseradish peroxidase-labeled antibody. After $1 \mathrm{~h}$ incubation at $37^{\circ} \mathrm{C}$, the plate was incubated with substrate A solution $(50 \mu \mathrm{L})$ and substrate B solution $(50 \mu \mathrm{L})$ at $37^{\circ} \mathrm{C}$ for $15 \mathrm{~min}$ in the dark. After adding $50 \mu \mathrm{L}$ of stopping solution, the $\mathrm{OD}$ value was measured at $450 \mathrm{~nm}$.

2.7. Differential Blood Cell Count. Blood samples collected from Experiment A were transferred to tubes containing EDTA (Sigma-Aldrich) as anticoagulant on Days 10, 15, 21, and 35 postimmunization. Blood samples collected from Experiment B were transferred to tubes containing EDTA as anticoagulant at 15 days postimmunization. A differential blood cell count was performed using an analyzer (Sysmex, pocH-100iV, JP). The numbers of neutrophil granulocytes, intermediate cells, and lymphocytes were counted.

2.8. Real-Time PCR (RT-PCR). RNA was extracted from spleen samples collected from Experiment $B$ at 30 days postimmunization using the PureLink RNA Mini Kit (Life Technologies, USA). The concentration of total RNA was determined by the OD value at $260 \mathrm{~nm}$. Reverse transcription was performed using a commercially available kit (Promega, 
IgG

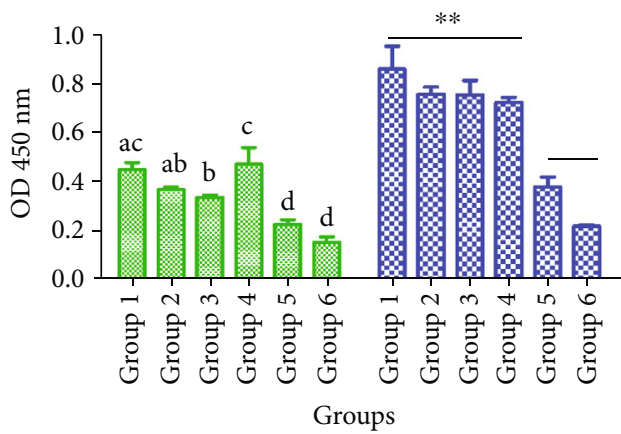

15 days post immunization

30 days post immunization
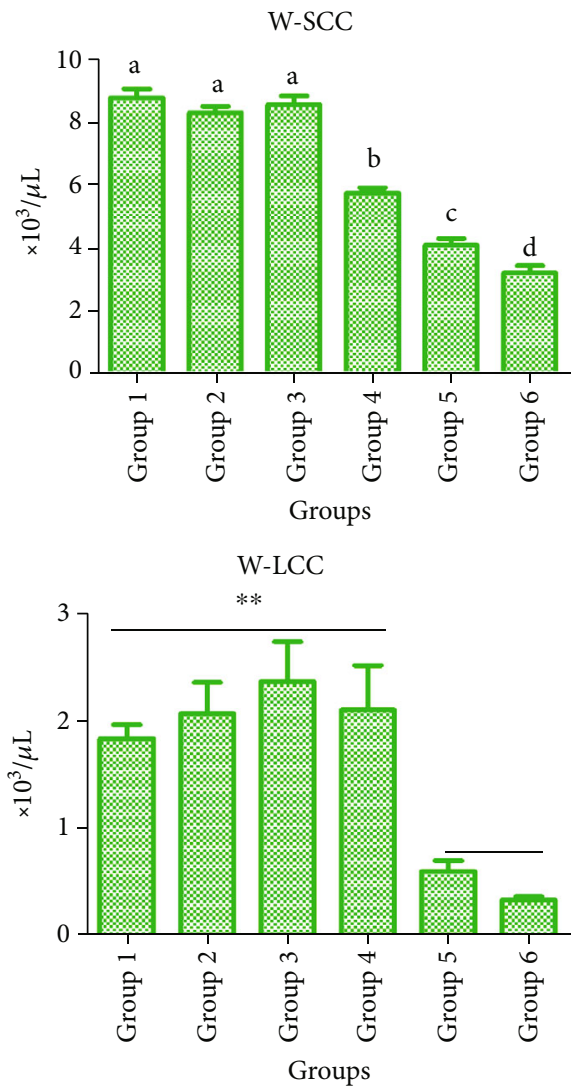
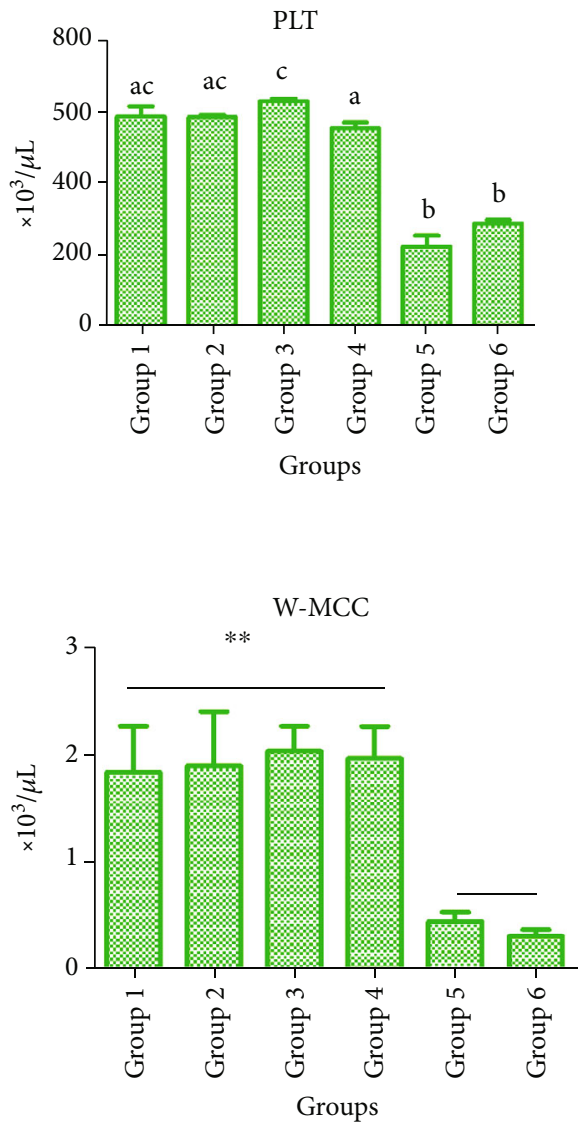

WBC-1

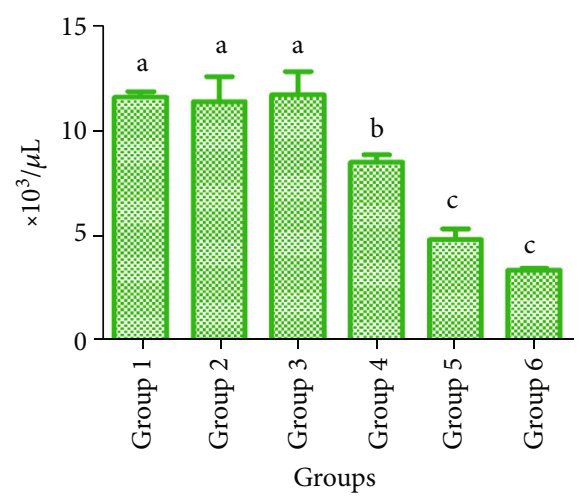

FIGURE 2: The serum level of whole bacterial protein-specific IgG antibody in rabbits from Experiment B was detected by indirect ELISA at 15 and 30 days postinjection. Blood samples were collected as anticoagulants at 15 days after injection for the detection of PLT, SCC, MCC, LCC, and $\mathrm{WBC}$.

Madison, WI, USA). Then, RT-PCR was performed to determine the expression levels of NF- $\kappa \mathrm{B} 2$ and TLR2. The primers used in this experiment (Table 3) were designed according to rabbit genome sequences shown in GenBank. ACTB was used as an internal control. The $2^{-\triangle \Delta C T}$ method was used for relative quantification. Each sample was analyzed at least twice.

2.9. Western Blot. Frozen spleen tissue samples were homogenized using RIPA buffer and then centrifuged at $12000 \mathrm{rpm}$ for $10 \mathrm{~min}$ at $4^{\circ} \mathrm{C}$ to obtain supernatants. Protein concentration was determined by the BCA method.
Samples were added with $5 \mathrm{x}$ SDS loading buffer, boiled at $100^{\circ} \mathrm{C}$ for $5 \mathrm{~min}$, separated on SDS-PAGE, and finally transfected to the PVDF membrane. After blocked with $5 \%$ milk at room temperature for $1 \mathrm{~h}$, the membrane was incubated with anti-TLR2 antibody $(1: 2000$, Cat No. NBP2-24909, Novus) and anti-beta-actin antibody $(1: 20000$, Cat No. 8226 , Abcam $)$ overnight at $4^{\circ}$ C. After three washes with PBST, the membrane was incubated with a goat anti-mouse secondary antibody $(1: 5000$, Cat No. 00001-1, Wuhan Sanying, China) $(1: 5000)$ at room temperature for $1 \mathrm{~h}$. The same amounts of enhanced luminol reagent and oxidizing reagent were diluted in $\mathrm{ddH}_{2} \mathrm{O}$ 


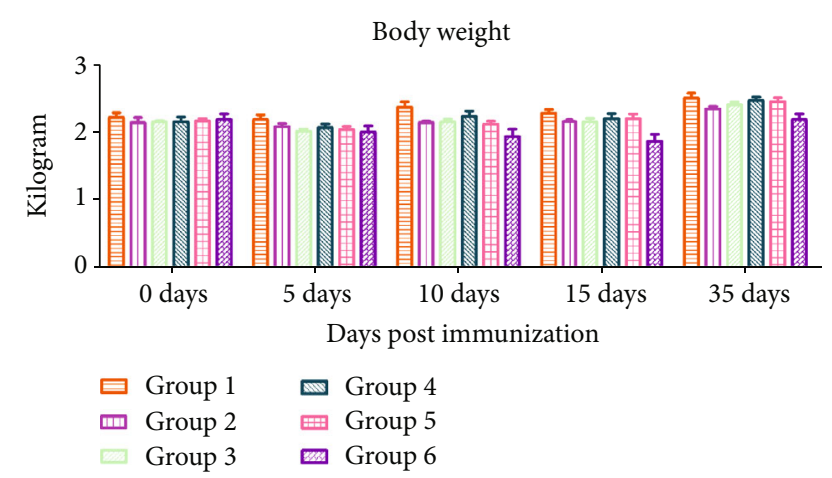

FIgure 3: The body weight of rabbits in Experiment A was measured before and after immunization on Days $0,5,10,15$, and 35 .

and then dropped on the sealing film. The results were analyzed using a gel imaging system.

2.10. Immunofluorescence Analysis of $N F-\kappa B$. HeLa cells were cultured in $10 \%$ serum medium in a $37^{\circ} \mathrm{C} / \mathrm{CO}_{2}$ incubator until $70 \%$ confluence. Then, cells were digested by trypsin, collected, and evenly plated into a 24 -well plate covered with aseptic climbing tablets. Rg1 (1 mg) was dissolved in $1 \mathrm{~mL}$ PBS, which was then diluted in gradient with culture medium. Cells were incubated with medium containing with or without Rg1 at 10, 20, 40, 100, 200, and $400 \mu \mathrm{g} / \mathrm{mL}$ for $2 \mathrm{~h}$. After treatment, cells were fixed with $4 \%$ paraformaldehyde for $20 \mathrm{~min}$, followed by $15 \mathrm{~min}$ incubation with $0.5 \%$ Triton $\mathrm{X}-100$ (prepared by $1 \mathrm{x}$ PBS) at room temperature. After washing, $50 \mu \mathrm{L}$ of diluted ProteinFind ${ }^{\circledR}$ anti-NF- $\kappa \mathrm{B}$ p65 mouse monoclonal antibody $(1: 100$, Cat No. HA106) was added to each cell climbing tablet and incubated at $4^{\circ} \mathrm{C}$ overnight. Subsequently, climbing tablets were gently washed with 1x PBST for $5 \mathrm{~min}$ and incubated with a fluorescent ProteinFind ${ }^{\circledR}$ secondary anti-rabbit IgG $(\mathrm{H}+\mathrm{L})$ antibody conjugated to AF488 (1:100, Cat No. HS131) at $37^{\circ} \mathrm{C}$ for $1 \mathrm{~h}$. After three washes with $1 \mathrm{x}$ PBST, samples were incubated with $40 \mu \mathrm{L}$ of Hoechst 33342 for 15 min to stain the nuclei. Then, the cell climbing sheet was sealed with sealing liquid and cells were photographed under a fluorescence microscope.

2.11. Statistical Analysis. Data were shown as the mean \pm SD. The differences among groups were compared using Tukey's HSD test and one-way ANOVA. A $P$ value of $<0.05$ was considered statistically significant.

\section{Results}

3.1. Measurements of the B. bronchiseptica-Specific Serum IgG Level and Antibody Agglutination. In Experiment A, the serum levels of $B$. bronchiseptica-specific IgG in rabbits after immunization were monitored over time (Figure 1) (SOD450nm). The IgG concentration started to increase at 5 days postinjection in all groups. From Days 10-21, the serum IgG level in rabbits immunized with $B$. bronchiseptica dissolved in Rg1-oil adjuvant was significantly increased com- pared to that in other adjuvant groups $(P<0.05)$. On Days 10 and 15, the serum IgG concentration in the oil group was significantly higher than that in other adjuvant groups $(P<0.05)$, except the Rg1-oil group.

Compared to other adjuvant groups, the antibody agglutination in animals immunized with B. bronchiseptica was significantly enhanced by Rg1-oil adjuvant and oil alone from Days 10 to $35(P<0.05)$. No significant difference was observed between the Rg1-oil and oil groups (Figure 1) (S$\mathrm{Bb}$ antibody agglutination).

In Experiment B, the serum levels of B. bronchisepticaspecific IgG in rabbits after immunization were monitored over time (Figure 2) (S-IgG). Compared to other adjuvant groups, the serum IgG level in rabbits immunized with $B$. bronchiseptica was effectively increased by $\operatorname{Rg} 1$ (100, 200, and $400 \mu \mathrm{g}$ ) plus oil and oil alone from Days 15 to 30 $(P<0.05)$.

3.2. Differential Blood Cell Count. In Experiment A, the number of WBC in rabbits immunized with B. bronchiseptica was significantly increased by $\mathrm{Rg} 1$-oil adjuvant compared to other adjuvant groups $(P<0.05)$ on Days 15 and 35 (Figure 1) (S-WBC cell detection). On Day 21, the number of SCC in rabbits immunized with B. bronchiseptica dissolved in Rg1-oil adjuvant was significantly increased compared to that in other adjuvant groups $(P<0.05$, Figure 1$)$ (S-SCC cell detection).

In Experiment B, the numbers of PLT, MCC, and LCC in rabbits immunized with $B$. bronchiseptica were significantly increased by $\operatorname{Rg} 1(100,200$, and $400 \mu \mathrm{g})$ plus oil and oil alone compared to other adjuvant groups $(P<0.05)$ on Day 15 (Figure 2) (S-PLT, S-W-MCC, and S-W-LCC). On Day 15, the numbers of SCC and WBC were also significantly increased by $\operatorname{Rg} 1(100,200$, and $400 \mu \mathrm{g})$ plus oil compared to other adjuvant groups $(P<0.05$, Figure 2$)$ (S-W-SCC, SWBC-1).

3.3. Effect of Rg1 on Growth Performance. The body weight of all groups was measured before and after immunization on Days $0,5,10,15$, and 35. No significant difference was observed among different groups (Figure 3) (S-Body weight).

3.4. Measurement of Cytokine Levels. The cytokine levels after immunization in Experiment B were monitored over time (Figure 4). The concentrations of IL-2 and IL-4 in the Rg1 $(100 \mu \mathrm{g})$ plus oil group were significantly higher than those in other adjuvant groups on Days 15 and $30 \quad(P<0.05$, Figure 4) (S-IL-2 15 days postimmunization; S-IL-2 35 days postimmunization; S-IL-4 15 days postimmunization; and S-IL-4 35 days postimmunization).

3.5. RT-PCR and Western Blot. Total RNA and protein were extracted from spleen tissue samples from rabbits in Experiment $B$ at 30 days postimmunization. The mRNA expression of NF- $\kappa \mathrm{B} 2$ in the oil group was significantly higher compared to that in other groups $(P<0.05)$ on Day 30 after immunization (Figure 5) (S-NF- $\kappa$ B RT-PCR). The protein levels of NF$\kappa \mathrm{B} 2$ in the $\mathrm{Rg} 1(100 \mu \mathrm{g})$ plus oil and antigen groups were significantly higher compared to those in others on Day 30 
IL-2

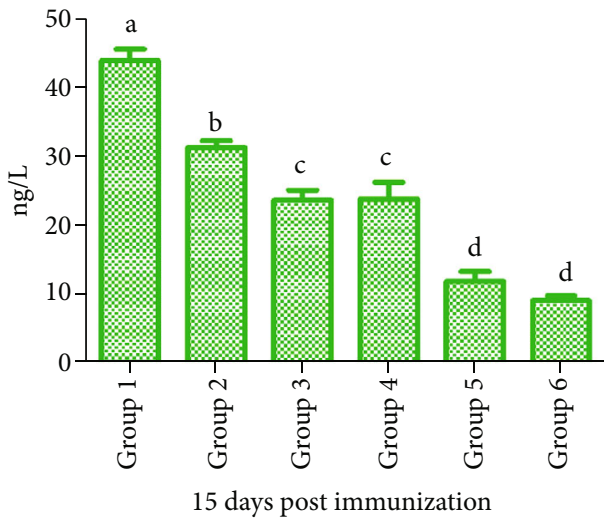

IL-4

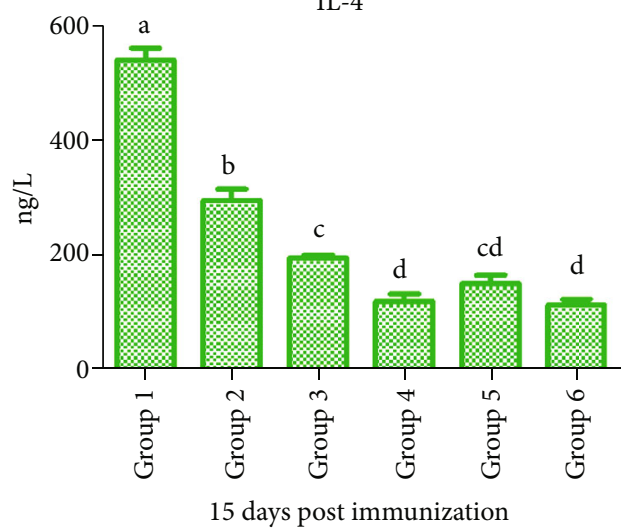

IL-2

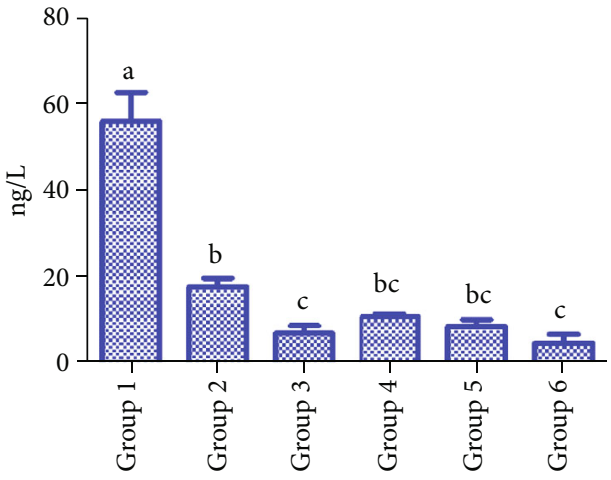

30 days post immunization

IL-4

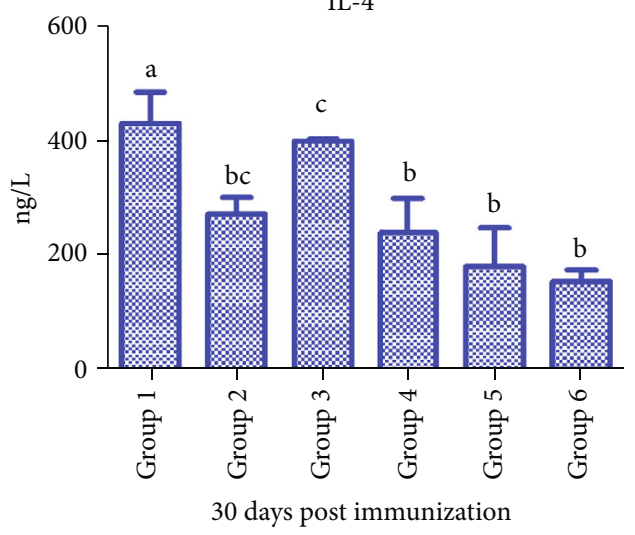

FIgURE 4: The levels of IL-2 and IL-4 in rabbits from Experiment B were assessed by ELISA at 15 and 30 days postimmunization.

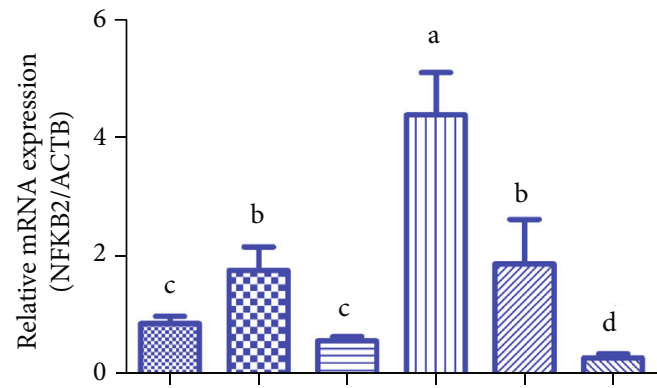

(a)

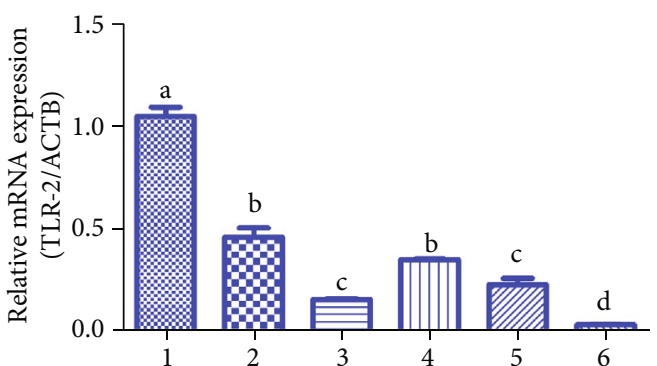

(b)

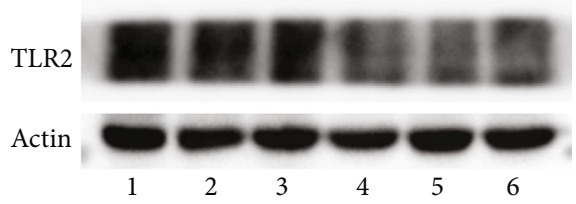

(c)

FIgURE 5: The mRNA and protein expressions of NF- $\kappa$ B2 and TLR2 in the spleen. Total RNA was isolated from the spleen samples collected at 30 days postimmunization. The spleen tissues were homogenized using RIPA buffer.

$(P<0.05)$, except the oil group (Figure 5(a)). The Rg1-oil $(100 \mu \mathrm{g})$ group showed significantly increased TLR2 expression on Day 30 compared with other groups $(P<0.05$, Figure 5(b)) (S-TLR2 RT-PCR). The protein level of TLR2 in the Rg1-oil $(100,200$, and $400 \mu \mathrm{g})$ group was also higher compared to that in other groups on Day 30 (Figure 5(c)).
3.6. Immunofluorescence Analysis of $N F-\kappa B$. The expression of NF- $\kappa \mathrm{B}$ in HeLa cells was dose-dependently increased in the presence of $\operatorname{Rg} 1 \quad(10-200 \mu \mathrm{g})$, while decreased when exposed to $\mathrm{Rg} 1$ at $400 \mu \mathrm{g}$ (Figure 6). The expression of Hoechst 33342 showed the same change when compared with the immunofluorescence staining of NF- $\kappa \mathrm{B}$ (Figure 6). 


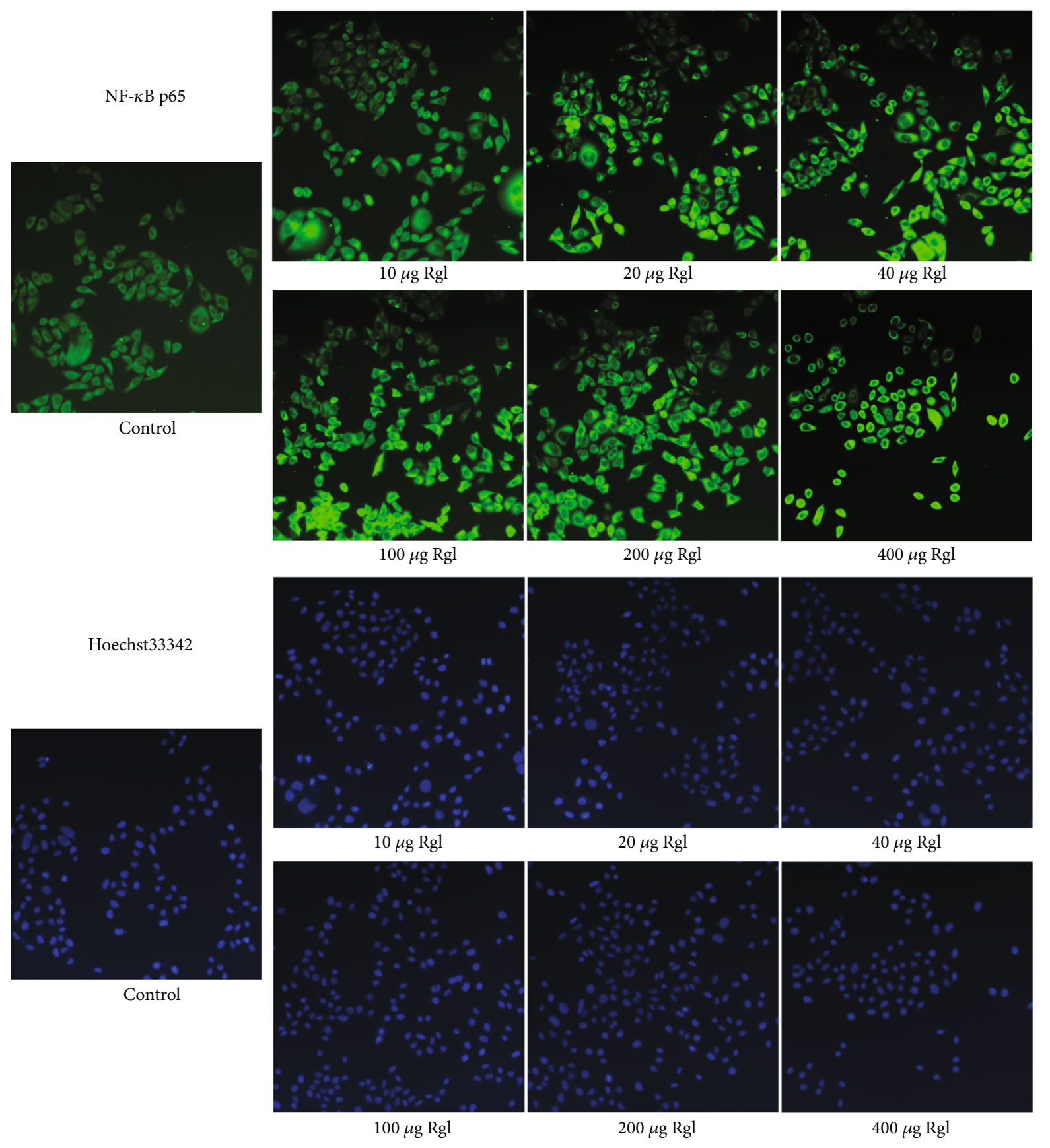

FIgURE 6: Rg1 (1 mg) was dissolved in $1 \mathrm{~mL}$ PBS and then diluted in gradient with culture medium. Cells were incubated with medium containing with or without Rg1 at 10,20, 40,100, 200, and $400 \mu \mathrm{g} / \mathrm{mL}$ for $2 \mathrm{~h}$.

\section{Discussion}

The rapid spread of $B$. bronchiseptica infection often leads to huge economic loss [8]. The application of attenuated $B$. bronchiseptica vaccines is limited due to safety issues during storage and transportation. Inactivated vaccines are safe, but the immunogenicity of $B$. bronchiseptica antigens is low [8]. Therefore, it is urgent to find an effective vaccine adjuvant for B. bronchiseptica vaccine. Since the original report by Espinet et al. [9] showing that saponins can be used as a vaccine adjuvant, considerable efforts have been expended to evaluate the adjuvant activity of saponins. On the one hand, saponin-based adjuvant stimulates cell-mediated immune responses and enhances antibody production at low doses. On the other hand, undesirable side effects caused by some saponins limited their application as vaccine adjuvants $[10,11]$. Panax ginseng is a tonic medicine commonly used in Asian countries. Rg1 extracted from ginseng shows low hemolytic activity [12] and high immunomodulating activity $[6,13]$ and therefore could be used as an adjuvant for veterinary vaccines.

The syringe extrusion method has a good background for immunization [14]. The water-in-oil emulsion is prepared using syringe extrusion, vortex, or high-speed 
homogenization for laboratory and clinical peptide-based vaccination. In this study, we showed a synergistic effect between $\operatorname{Rg} 1$ and oil, consistent with previous studies showing that a mixture of saponins and oil improved the immune effect of vaccines $[15,16]$. We also evaluated the effects of other adjuvants on inactivated $B$. bronchiseptica vaccine. The results indicated that ELISA was more sensitive than the agglutination test. The serum antibody level began to increase in all groups of rabbits since Day 5 postimmunization. The Rg1 $(400 \mu \mathrm{g})$ plus oil group showed a significantly increased antibody level and antibody titer compared to other groups, indicating the synergistic effect of Rg1-oil adjuvant on boosting humoral responses in rabbits. Moreover, the cellular immune test showed that Rg1, Rg1-oil, oil, and QA groups had a higher number of WBC compared to the aluminum glue and control groups since Day 10 postimmunization, but no significant difference was observed among Rg1, Rg1-oil, oil, and QA groups. At 15 and 35 days postimmunization, the number of WBC in the Rg1-oil group was significantly higher than that in other groups, suggesting that Rg1-oil adjuvant significantly improved cellular immune responses in rabbits. At 21 days postimmunization, the Rg1-oil group showed a significantly higher number of SCC compared to other groups. On Day 35 after immunization, the Rg1-oil group showed significantly more SCC than other groups (except the Rg1 group). Previous evidence demonstrated that Rg1 increased the proportion of $\mathrm{T}$ helper cells among all $\mathrm{T}$ cells and promoted the expression of IL-4 and IL-2 in murine splenocytes [17]. In Experiment B, the levels of IL-4 and IL-2 in the Rg1 $(100 \mu \mathrm{g})$ plus oil group were significantly higher than those in other groups, while no significant difference was observed between the oil group and other groups, indicating that $\mathrm{Rg} 1$ in $\mathrm{Rg} 1$-oil adjuvant promoted the production of IL-4 and IL-2 in immunized rabbits.

Rg1 usually exerts an inhibitory effect on NF- $\kappa$ B in inflammation. For example, Rg1 extracted from white ginseng plays an anti-inflammatory role by reducing the expression of NF- $\kappa \mathrm{B}$ [18]. It was also found that Rg1 effectively suppressed allergic airway inflammation of asthma partly through the TNF- $\alpha / N F-\kappa B$ pathway [19]. Our results showed that the expression of NF- $\kappa$ B in the Rg1 (100-400 $\mu \mathrm{g})$ plus oil group was significantly lower than that in the oil group, implying that Rg1-oil adjuvant downregulated NF- $\kappa \mathrm{B}$ in noninflammatory condition. The in vitro study demonstrated that Rg1 promoted the expression of NF- $\kappa \mathrm{B}$ in HeLa cells at doses between $10 \mu \mathrm{g}$ and $200 \mu \mathrm{g}$ but inhibited NF- $\kappa \mathrm{B}$ expression at $400 \mu \mathrm{g}$, suggesting that $\mathrm{Rg} 1$ had an inhibitory effect on HeLa cells. These findings were also consistent with the RT-PCR results, which showed the splenic expression of NF- $\kappa \mathrm{B}$ in the $\operatorname{Rg} 1$ (100-400 $\mu \mathrm{g})$ plus oil group. TLRs are key transmembrane pattern recognition receptors that play an important role in initiating immune responses against invading microbial pathogens. Our data showed that $\operatorname{Rg} 1(100 \mu \mathrm{g})$ plus oil significantly increased the expression of TLR2 in splenocytes, which was consistent with a previous report [20]. It has also been shown that active components of Panax ginseng possess a putative TLR ligand in different cell models $[21,22]$.

\section{Conclusion}

$\operatorname{Rg} 1(100 \mu \mathrm{g})$ plus oil significantly improved the immune effect of rabbit B. bronchiseptica vaccine at both the humoral and cellular levels. Rg1-oil adjuvant increased the production of IL-2 and IL-4 in rabbits after immunization. Rg1 $(100 \mu \mathrm{g})$ plus oil significantly upregulated TLR2 expression but decreased the level of NF- $\kappa$ B in splenocytes. Rg1-oil adjuvant may increase the levels of IL- 2 and IL- 4 by upregulating TLR2, thus enhancing the immune effect of B. bronchiseptica vaccine. Thus, Rg1-oil adjuvant could be used as a potential vaccine adjuvant for rabbit $B$. bronchiseptica vaccine.

\section{Data Availability}

Data could be found in supplemental files.

\section{Conflicts of Interest}

The authors declare no conflict of interest.

\section{Authors' Contributions}

Xiao Chenwen and Ji Quanan make equal contributions in the MS.

\section{Acknowledgments}

This study was supported by the National Natural Science Foundation (31402241), the Basic Public Welfare Research Projects in Zhejiang Province (LGN18C180003), and the National Rabbit Industry Project of China (nycytx-44-3-2).

\section{Supplementary Materials}

Concise supplementary material description: W-SCC: in Experiment B (Figure 2). W-MCC: in Experiment B (Figure 2). W-LCC: in Experiment B (Figure 2). WBC-1: in Experiment B (Figure 2). SCC cell detection: in Experiment A (Figure 1). PLT: in Experiment B (Figure 2). OD450nm: in Experiment A (Figure 1). IL-4 35 days postimmunization: in Experiment B (Figure 4). IL-2 35 days postimmunization: in Experiment B (Figure 4). Body weight: in Experiment A (Figure 3). IL-4 15 days postimmunization: in Experiment B (Figure 4). IL-2 15 days postimmunization: in Experiment B (Figure 4). IgG: in Experiment B (Figure 2). WBC cell detection: in Experiment A (Figure 1). Bb antibody agglutination: in Experiment A (Figure 1). (Supplementary Materials)

\section{References}

[1] C. Garcia-de-la-Fuente, L. Guzman, M. E. Cano et al., "Microbiological and clinical aspects of respiratory infections associated with _Bordetella bronchiseptica_" Diagnostic Microbiology and Infectious Disease, vol. 82, no. 1, pp. 20-25, 2015.

[2] S. Pruller, U. Rensch, D. Meemken et al., "Antimicrobial susceptibility of Bordetella bronchiseptica isolates from swine and companion animals and detection of resistance genes," PLoS One, vol. 10, no. 8, article e0135703, 2015. 
[3] Y. Liu, H. Chen, Q. Wei, C. Xiao, Q. Ji, and G. Bao, "Immune efficacy of five novel recombinant Bordetella bronchiseptica proteins," BMC Veterinary Research, vol. 11, no. 1, p. 173, 2015.

[4] A. C. Yendo, F. de Costa, S. P. Cibulski et al., "A rabies vaccine adjuvanted with saponins from leaves of the soap tree (Quillaja brasiliensis) induces specific immune responses and protects against lethal challenge," Vaccine, vol. 34, no. 20, pp. 2305-2311, 2016.

[5] F. Su, Y. Xue, Y. Wang, L. Zhang, W. Chen, and S. Hu, "Protective effect of ginsenosides Rg1 and Re on lipopolysaccharideinduced sepsis by competitive binding to Toll-like receptor 4," Antimicrobial Agents and Chemotherapy, vol. 59, no. 9, pp. 5654-5663, 2015.

[6] J. Sun, S. Hu, and X. Song, "Adjuvant effects of protopanaxadiol and protopanaxatriol saponins from ginseng roots on the immune responses to ovalbumin in mice," Vaccine, vol. 25, no. 6, pp. 1114-1120, 2007.

[7] F. Su, L. Yuan, L. Zhang, and S. Hu, "Ginsenosides Rg1 and Re act as adjuvant via TLR4 signaling pathway," Vaccine, vol. 30, no. 27, pp. 4106-4112, 2012.

[8] C. Xiao, G. Bao, Y. Liu et al., "Greater efficacy of the ECMS-oil adjuvant over other formulations on immune responses against Bordetella bronchiseptica in rabbits and the underlying mechanism," International Immunopharmacology, vol. 38, pp. 194-203, 2016.

[9] G. P. Rédei, "Saponins," in Encyclopedia of Genetics, Genomics, Proteomics and Informatics, p. 1755, Springer Science \& Business Media, Netherlands, Dordrecht, 2008.

[10] Z.-G. Yang, H.-X. Sun, and W.-H. J. V. Fang, "Haemolytic activities and adjuvant effect of Astragalus membranaceus saponins (AMS) on the immune responses to ovalbumin in mice," Vaccine, vol. 23, no. 44, pp. 5196-5203, 2005.

[11] H.-X. Sun and H.-J. J. V. Pan, "Immunological adjuvant effect of Glycyrrhiza uralensis saponins on the immune responses to ovalbumin in mice," Vaccine, vol. 24, no. 11, pp. 1914-1920, 2006.

[12] D.-y. Lee, B.-s. Choi, I.-h. Lee, J.-h. Kim, and P.-s. Gwon, "Comparison of index compounds content and antioxidative activity of wild ginseng pharmacopuncture by extraction methods," The Journal of Internal Korean Medicine, vol. 39, no. 3, pp. 313-322, 2018.

[13] B. Kenarova, H. Neychev, C. Hadjiivanova, and V. D. Petkov, "Immunomodulating activity of ginsenoside Rg1 from Panax ginseng," Japanese Journal of Pharmacology, vol. 54, no. 4, pp. 447-454, 1990.

[14] Y. T. Koh, S. A. Higgins, J. S. Weber, and W. M. Kast, "Immunological consequences of using three different clinical/laboratory techniques of emulsifying peptide-based vaccines in incomplete Freund's adjuvant," Journal of Translational Medicine, vol. 4, no. 1, pp. 1-12, 2006.

[15] X. Song, S. Bao, L. Wu, and S. J. V. Hu, "Ginseng stem-leaf saponins (GSLS) and mineral oil act synergistically to enhance the immune responses to vaccination against foot-and-mouth disease in mice," Vaccine, vol. 27, no. 1, pp. 51-55, 2009.

[16] C. Xiao, Z. I. Rajput, and S. J. V. Hu, "Improvement of a commercial foot-and-mouth disease vaccine by supplement of Quil A," Vaccine, vol. 25, no. 25, pp. 4795-4800, 2007.

[17] E.-j. Lee, E. Ko, J. Lee et al., "Ginsenoside Rg1 enhances CD4 ${ }^{+}$ T-cell activities and modulates Th1/Th2 differentiation," International Immunopharmacology, vol. 4, no. 2, pp. 235244, 2004.
[18] M. He, X. Huang, S. Liu et al., "The difference between white and red ginseng: variations in ginsenosides and immunomodulation," Planta Medica, vol. 84, pp. 845-854, 2018.

[19] K. Xue, L. Ruan, J. Hu, Z. Fu, D. Tian, and W. Zou, “Panax notoginseng saponin R1 modulates TNF- $\alpha / N F-\kappa B$ signaling and attenuates allergic airway inflammation in asthma," International Immunopharmacology, vol. 88, p. 106860, 2020.

[20] C. Baravalle, P. Silvestrini, M. C. Cadoche et al., "Intramammary infusion of_Panax ginseng_extract in bovine mammary gland at cessation of milking induces changes in the expression of toll-like receptors, MyD88 and NF-kB during early involution," Research in Veterinary Science, vol. 100, pp. 52-60, 2015.

[21] M. Pannacci, V. Lucini, F. Colleoni et al., "Panax ginseng C.A. Mayer G115 modulates pro-inflammatory cytokine production in mice throughout the increase of macrophage toll-like receptor 4 expression during physical stress," Brain, Behavior, and Immunity, vol. 20, no. 6, pp. 546-551, 2006.

[22] T.-A. Nakaya, M. Kita, H. Kuriyama, Y. Iwakura, and J. Imanishi, "Panax ginseng induces production of proinflammatory cytokines via toll-like receptor," Journal of Interferon \& Cytokine Research, vol. 24, no. 2, p. 93, 2004. 